\title{
Clinical significance of histological classification of idiopathic interstitial pneumonia
}

\author{
K.R. Flaherty*, G.B. Toews*, W.D. Travis ${ }^{\#}$, T.V. Colby ${ }^{\Uparrow}$, E.A. Kazerooni ${ }^{+}$, B.H. Gross ${ }^{+}$, A. Jain ${ }^{\S}$, \\ R.L. Strawderman III ${ }^{\S}$ R. Paine*, A. Flint ${ }^{f}$, J.P. Lynch III*, F.J. Martinez*
}

Clinical significance of histological classification of idiopathic interstitial pneumonia. K.R. Flaherty, G.B. Toews, W.D. Travis, T.V. Colby, E.A. Kazerooni, B.H. Gross, A. Jain, R.L. Strawderman III, R. Paine, A. Flint, J.P. Lynch III, F.J. Martinez. (C)ERS Journals Ltd 2002.

ABSTRACT: Patients with idiopathic interstitial pneumonias (IIPs) can be subdivided into groups based on the histological appearance of lung tissue obtained by surgical biopsy. The quantitative impact of histological diagnosis, baseline factors and response to therapy on survival has not been evaluated.

Surgical lung biopsy specimens from 168 patients with suspected IIP were reviewed according to the latest diagnostic criteria. The impact of baseline clinical, physiological, radiographic and histological features on survival was evaluated using Cox regression analysis. The predictive value of honeycombing on high-resolution computed tomography (HRCT) as a surrogate marker for usual interstitial pneumonia (UIP) was examined. The response to therapy and survival of 39 patients treated prospectively with high-dose prednisone was evaluated.

The presence of UIP was the most important factor influencing mortality. The risk ratio of mortality when UIP was present was 28.46 (95\% confidence interval (CI) $5.5-148.0 ; p=0.0001$ ) after controlling for patient age, duration of symptoms, radiographic appearance, pulmonary physiology, smoking history and sex. Honeycombing on HRCT indicated the presence of UIP with a sensitivity of $90 \%$ and specificity of $86 \%$. Patients with nonspecific interstitial pneumonia were more likely to respond or remain stable (9 of 10$)$ compared to patients with UIP (14 of 29) after treatment with prednisone. Patients remaining stable had the best prognosis. The risk ratio of mortality for stable patients compared to nonresponders was $0.32(95 \%$ CI $0.11-0.93$; $\mathrm{p}=0.04)$ in all patients and $0.33(95 \%$ CI $0.12-0.96 ; p=0.04)$ in patients with UIP.

The histological diagnosis of usual interstitial pneumonia is the most important factor determining survival in patients with suspected idiopathic interstitial pneumonia. The presence of honeycombing on high-resolution computed tomography is a good surrogate for usual interstitial pneumonia and could be utilized in patients unable to undergo surgical lung biopsy. Patients with nonspecific interstitial pneumonia are more likely to respond or remain stable following a course of prednisone. Patients remaining stable following prednisone therapy have the best prognosis.

Eur Respir J 2002; 19: 275-283.

\begin{abstract}
*Division of Pulmonary and Critical Care Medicine and Depts of ${ }^{+}$Radiology and ${ }^{\text {Pathology, University of }}$ Michigan Health System, Ann Arbor, MI, "Armed Forces Institute of Pathology, Washington DC, "Mayo Clinic, Scottsdale, AZ, and ${ }^{8}$ Dept of Biostatistics, University of Michigan School of Public Health, Ann Arbor, MI, USA.
\end{abstract}

Correspondence: F.J. Martinez

Taubman Center 3916

1500 E. Medical Center Dr.

Ann Arbor, MI 48109-0360

USA

Fax: 17349365048

E-mail: fmartine@umich.edu

Keywords: Idiopathic pulmonary fibrosis

nonspecific interstitial pneumonia usual interstitial pneumonia

Received: February 232001

Accepted after revision September 6 2001

This study was supported in part by National Institutes of Health (NIH) National Heart, Lung, and Blood Institute (NHLBI) Grant \#P50HL46487, NIH/NCRR 3 MO1 RR00042-33S3, NIH/NIA P60 AG08808-06 and NHLBI, 1 K24 HL04212-01.
A recent consensus statement has proposed that idiopathic interstitial pneumonias (IIPs) be divided into histopathological subsets that differ in prognosis and response to therapy [1]. These subsets include usual interstitial pneumonia (UIP), nonspecific interstitial pneumonia (NSIP), respiratory bronchiolitis interstitial lung disease (RBILD), desquamative interstitial pneumonia (DIP), and others $[2,3]$. This approach, by definition, requires surgical lung biopsy (SLB).

The clinical features of IIP are nonspecific. Reliable noninvasive markers of the above pathological subsets or of clinical outcome are lacking. In the present study, a large group of patients with IIP were prospectively evaluated and categorized into histopathological subsets using the latest histopathological criteria. The purpose of the study was to: 1) quantify the role of histopathological classification in predicting survival while adjusting for baseline demographic, physiological and radiographic factors; 2) evaluate the utility of semiquantitative high-resolution computed tomography (HRCT) in predicting survival; and 3) evaluate the response to high-dose prednisone for a subset of patients with NSIP and UIP treated prospectively using a standardized protocol and the influence of response on survival.

\section{Methods}

\section{Patient selection}

SLB specimens from patients suspected of having IIP referred by participants in the University of Michigan Fibrotic Lung Disease Network between 
October 1989 and February 2000 were reviewed by three pathologists. In all patients, the suspicion of IIP was based on symptoms and physiological and radiological findings. None had been previously treated for interstitial lung disease. A subgroup $(n=39)$ of these patients categorized as having IPF using previously available criteria [4] had been reported [5, 6]. Using the latest histopathological criteria (see below), 14 of these patients were classified as having a histological pattern other than that of UIP.

\section{Baseline characterization}

A baseline assessment of severity of dyspnoea was made using the level of dyspnea (LOD) scale [7], the baseline dyspnea index (BDI) [8] and the oxygen consumption diagram [9].

Physiological testing. Physiological assessment was performed before SLB and initiation of therapy. Pulmonary function tests including spirometry and measurement of lung volumes and diffusion capacity of the lung for carbon monoxide were performed on the same day and before a cardiopulmonary exercise test [6].

Clinical, radiological and physiological scoring. Overall clinical severity was assessed using a previously developed clinical, radiological and physiological (CRP) composite score [7]. The total CRP score ranges $0-100$ points ( 100 being the most severe disease) based on variables including the LOD scale, chest radiography, pulmonary function test results, and resting and exercise oxygenation levels (exercise component). A physiological component (PHYSIO) was constructed by incorporating the scores from pulmonary function and resting oxygenation level.

High-resolution computed tomography. HRCT examinations were performed using 1.0 - or $1.5-\mathrm{mm}$-thick sections taken at $1-\mathrm{cm}$ intervals throughout the entire lungs during inspiration in the supine position and through the caudal $10 \mathrm{~cm}$ of the lungs at $2-3-\mathrm{cm}$ increments in the prone position. Two thoracic radiologists prospectively and independently scored all lobes on HRCT for ground-glass opacity (CTalv) and interstitial opacity (CTfib) on a scale of $0-5$; the mean score for each lobe and for the entire lung was calculated [10].

Pathological review of specimens. SLB specimens were reviewed by three pathologists blinded to their clinical features from March 1999 to February 2000. Each specimen was assigned a histological diagnosis using defined criteria $[2,11]$. Patients with NSIP were further classified as having predominantly cellular or fibrosing patterns $[2,11]$. UIP was defined by a patchy dense interstitial fibrosis, often with a subpleural and paraseptal distribution associated with loss of lung architecture, frequent honeycomb change and fibroblastic foci [2]. Interstitial inflammation was usually minimal. Fibrotic NSIP was defined by a dense or loose interstitial fibrosis lacking the temporal heterogeneity of UIP and usually showing diffuse uniform involvement of the lung tissue (spatial uniformity). Fibroblastic foci were inconspicuous or absent and inflammation was mild to moderate. Cellular NSIP was characterized by mild-to-moderate chronic interstitial inflammation without interstitial fibrosis. Type II pneumocyte hyperplasia was often present in areas of inflammation. Foci of organizing pneumonia could be seen in both NSIP groups but these were focal and not the predominant feature. Cellular NSIP corresponded to group 1 of KATZENSTEIN and FIORELLI [12], whereas fibrotic NSIP corresponded to groups 2 and 3. Acknowledging that the definition of NSIP was still evolving at the beginning of the study, all specimens classified as NSIP were rereviewed at the end of the study period for consistency and to confirm the accuracy of the initial diagnosis.

\section{Therapy}

A subgroup of patients with NSIP $(n=10)$ and UIP $(n=29)$ were treated using a protocol evaluating the effect of high-dose prednisone treatment in patients with pulmonary fibrosis. These patients received an initial course of high-dose prednisone $(1 \mathrm{mg} \cdot \mathrm{kg}$ ideal body weight ${ }^{-1} \cdot$ day $^{-1}$ up to $100 \mathrm{mg} \cdot \mathrm{day}^{-1}$ or $1 \mathrm{mg} \cdot \mathrm{kg}$ ideal body weight ${ }^{-1} \cdot$ day $^{-1}$ up to $60 \mathrm{mg}$ for the first month followed by $40 \mathrm{mg} \cdot \mathrm{day}^{-1}$ for the subsequent 2 months). Change in CRP score at 3 months was used to assess therapeutic response; responders demonstrated a $>10$-point drop in CRP score, stable an absolute change of $<10$ points and nonresponders a $>10$-point rise. Prednisone therapy was continued (in a tapering dose) in patients responding to initial therapy. In others, the prednisone was rapidly tapered and discontinued within 4 weeks and a 6-month course of oral cyclophosphamide $(n=14)$ or azathioprine $(n=11)$ prescribed. Five patients died prior to institution of subsequent therapy. The treatment regimens for patients with UIP, NSIP and RBILD/ DIP are listed in table 1, including a subset treated in a phase I trial comparing zileuton to azathioprine conducted at the University of Michigan, Ann Arbor, MI, USA.

\section{Statistical analysis}

Categorical data were compared between groups using the Fisher exact test, whereas continuous data were compared using analysis of variance (ANOVA). When ANOVA results indicated that groups differed, post hoc comparisons were performed using unpaired two-tailed t-tests or a Wilcoxon rank-sum test for nonparametric data. All t-tests assumed unequal variances and a p-value of $<0.05$ was considered significant. Where appropriate, data are expressed as mean + SD. The Fisher exact test was used when comparing the distribution of responder/stable/nonresponder status across UIP and NSIP groups. Survival was illustrated via Kaplan-Meier curves. Cox regression analysis was used to examine the 
Table 1.-Treatment administered to 161 patients with usual interstitial pneumonia (UIP), nonspecific interstitial pneumonia (NSIP) and respiratory bronchiolitis interstitial lung disease/desquamative interstitial pneumonia (RBILD/ DIP)

\begin{tabular}{|c|c|c|c|}
\hline Treatment regimen & $\begin{array}{l}\text { UIP } \\
\text { n }(\%)\end{array}$ & $\begin{array}{l}\text { NSIP } \\
\text { n }(\%)\end{array}$ & $\begin{array}{l}\text { RBILD/DIP } \\
\mathrm{n}(\%)\end{array}$ \\
\hline None & $7(7)$ & $2(6)$ & $2(9)$ \\
\hline Prednisone alone* & $35(33)$ & $18(55)$ & $10(46)$ \\
\hline $\begin{array}{l}\text { Prednisone+azathioprine } \\
\text { or cyclophosphamide }\end{array}$ & $31(29)$ & $4(12)$ & $6(27)$ \\
\hline Zileuton & $21(20)$ & $4(12)$ & $0(0)$ \\
\hline Azathioprine alone & $5(4)$ & $4(12)$ & $1(4)$ \\
\hline Miscellaneous & $7(7)$ & $1(3)$ & $3(14)$ \\
\hline
\end{tabular}

*: 39 of the patients with UIP and NSIP received prednisone as part of a prospective study of high-dose steroids in idiopathic pulmonary fibrosis (see Therapy section for details).

relationship between histopathological subclassification and mortality, controlling for other potential confounders including patient age, duration of symptoms prior to biopsy, sex, smoking status, physiological parameters, including the CRP score and its components, and HRCT scores. As there were no deaths in the cellular NSIP group, all patients with NSIP were grouped for survival analyses.

\section{Results}

The histological diagnoses in the 168 patients reviewed were UIP $(n=106)$, fibrotic NSIP $(n=28)$, cellular NSIP $(n=5)$, RBILD/DIP $(n=22)$, hypersensitivity pneumonia $(\mathrm{n}=5)$ and bronchiolitis obliterans with organizing pneumonia $(n=1)$ plus one which remained unclassified. The baseline characteristics of the largest histological groups are shown in table 2. A baseline CRP score was available for 103 of these patients (table 3 ).

The mean follow-up periods for each group were as follows: UIP 1.94 yrs (range 0.1-6.98), fibrotic NSIP 2.82 yrs (range 0.30-8.16), cellular NSIP 4.77 yrs (range 0.42-6.72) and RBILD/DIP 3.58 yrs (range 0.8-7.59). There were $54(51 \%)$ deaths among UIP patients, three $(10 \%)$ among fibrotic NSIP patients, none among cellular NSIP patients and one among those with RBILD/DIP. Survival curves comparing UIP, NSIP and RBILD/DIP cases are shown in figure 1 . The most important predictor of survival was the absence of UIP. Using univariate

Table 2. - Baseline characteristics in the major histopathological categories

\begin{tabular}{|c|c|c|c|c|c|}
\hline Characteristic & $\begin{array}{c}\text { UIP } \\
\text { mean } \pm \text { SD }\end{array}$ & $\begin{array}{c}\text { Fibrotic NSIP } \\
\text { mean } \pm \text { SD }\end{array}$ & $\begin{array}{c}\text { Cellular NSIP } \\
\text { mean } \pm \mathrm{SD}\end{array}$ & $\begin{array}{c}\mathrm{RBILD} / \mathrm{DIP} \\
\text { mean } \pm \mathrm{SD}\end{array}$ & $\begin{array}{l}\text { ANOVA* } \\
\text { p-value }\end{array}$ \\
\hline Sex male/female & $50 / 56$ & $16 / 12$ & $3 / 2$ & $13 / 9$ & $0.73^{\#}$ \\
\hline Age at biopsy yrs & $62 \pm 11$ & $56 \pm 11$ & $50 \pm 9$ & $49 \pm 10$ & $<0.0001$ \\
\hline Time since symptom onset yrs & $2.8 \pm 2.9$ & $2.2 \pm 3.4$ & $1.8 \pm 2.0$ & $2.8 \pm 2.4$ & 0.52 \\
\hline Weight $\mathrm{kg}$ & $86 \pm 18$ & $87 \pm 18$ & $85 \pm 19$ & $86 \pm 18$ & 0.99 \\
\hline Smokers $\%$ & 60 & 71 & 40 & 59 & $0.51^{\#}$ \\
\hline Cigarette consumption pack-yrs & $18 \pm 22$ & $24 \pm 25$ & $7 \pm 14$ & $22 \pm 26$ & 0.32 \\
\hline \multicolumn{6}{|l|}{ Dyspnoea } \\
\hline LOD & $10 \pm 5$ & $10 \pm 4$ & $5 \pm 4$ & $14 \pm 4$ & 0.18 \\
\hline BDI & $6 \pm 2$ & $5 \pm 2$ & $8 \pm 3$ & NA & 0.36 \\
\hline OCD & $48 \pm 19$ & $46 \pm 18$ & $67 \pm 25$ & NA & 0.34 \\
\hline \multicolumn{6}{|l|}{ Spirometry } \\
\hline FVC L & $2.49 \pm 0.93$ & $2.68 \pm 0.59$ & $2.81 \pm 1.47$ & $2.91 \pm 0.98$ & 0.40 \\
\hline$\%$ pred & $67 \pm 19$ & $73 \pm 15$ & $72 \pm 24$ & $78 \pm 20$ & 0.18 \\
\hline FEV1 L & $2.04 \pm 0.73$ & $2.10 \pm 0.49$ & $2.24 \pm 0.94$ & $2.16 \pm 0.77$ & 0.87 \\
\hline$\%$ pred & $77 \pm 21$ & $78 \pm 19$ & $78 \pm 22$ & $78 \pm 26$ & 0.99 \\
\hline TLC L & $4.05 \pm 1.19$ & $4.47 \pm 1.09$ & $4.68 \pm 2.19$ & $5.18 \pm 1.51$ & 0.02 \\
\hline$\%$ pred & $73 \pm 16$ & $78 \pm 19$ & $78 \pm 22$ & $78 \pm 26$ & 0.02 \\
\hline$D \mathrm{~L}, \mathrm{CO} \mathrm{mL} \cdot \mathrm{min}^{-1} \cdot \mathrm{mmHg}^{-1}$ & $12.8 \pm 5.2$ & $11.8 \pm 3.2$ & $16.9 \pm 4.9$ & $16.4 \pm 3.1$ & 0.01 \\
\hline$\%$ pred & $51 \pm 17$ & $51 \pm 14$ & $69 \pm 14$ & $63 \pm 14$ & 0.02 \\
\hline \multicolumn{6}{|l|}{ HRCT Score } \\
\hline Alveolar & $1.85 \pm 0.90$ & $2.62 \pm 1.29$ & $1.60 \pm 2.10$ & $1.62 \pm 1.21$ & 0.03 \\
\hline Interstitial & $1.90 \pm 0.72$ & $0.87 \pm 0.62$ & $0.44 \pm 0.41$ & $0.63 \pm 0.42$ & $<0.0001$ \\
\hline Total & $3.75 \pm 0.96$ & $3.49 \pm 1.47$ & $2.04 \pm 2.04$ & $2.54 \pm 0.97$ & $<0.0001$ \\
\hline
\end{tabular}

*: the following post hoc comparisons were significant at the $\mathrm{p}=0.05$ level; all other comparisons were nonsignificant: usual interstitial pneumonia (UIP) versus fibrotic nonspecific interstitial pneumonia (NSIP), cellular NSIP and respiratory bronchiolitis interstitial lung disease/desquamative interstitial pneumonia (RBILD/DIP) and fibrotic NSIP versus RBILD/DIP (age); UIP versus RBILD/DIP (total lung capacity (TLC)); UIP versus fibrotic NSIP and RBILD/DIP (TLC, percentage of the predicted value (\% pred)); UIP versus RBILD/DIP and fibrotic NSIP versus cellular NSIP and RBILD/DIP (diffusion capacity of the lung for carbon monoxide (DL,CO)); UIP versus cellular NSIP and RBILD/DIP and fibrotic NSIP versus cellular NSIP and RBILD/DIP (DL,CO, \% pred); fibrotic NSIP versus UIP and RBILD/DIP (high-resolution) computed tomography score (HRCT score, alveolar) [10]; UIP versus fibrotic NSIP, cellular NSIP and RBILD/DIP (HRCT, interstitial); and UIP versus fibrotic NSIP, cellular NSIP and RBILD/DIP (HRCT, total). " : Fisher exact test. ANOVA: analysis of variance; LOD: level of dyspnea [scale] [7]; BDI: Baseline dyspnea index [8]; OCD: oxygen consumption diagram [9]; FVC: forced vital capacity; FEV1: forced expiratory volume in one second; NA: not available. 
Table 3. - Baseline clinical, radiological and physiological (CRP) score components in patients with idiopathic interstitial pneumonia (IIP) $(n=103)$

\begin{tabular}{lccccc}
\hline Characteristic & $\begin{array}{c}\text { UIP } \\
\text { mean } \pm \text { SD }\end{array}$ & $\begin{array}{c}\text { Fibrotic NSIP } \\
\text { mean } \pm \text { SD }\end{array}$ & $\begin{array}{c}\text { Cellular NSIP } \\
\text { mean } \pm \text { SD }\end{array}$ & $\begin{array}{c}\text { RBILD/DIP } \\
\text { mean } \pm \text { SD }\end{array}$ & $\begin{array}{c}\text { ANOVA* } \\
\text { p-value }\end{array}$ \\
\hline LOD & $10 \pm 5$ & $10 \pm 4$ & $5 \pm 4$ & $14 \pm 4$ & 0.18 \\
A-a rest & $21 \pm 13$ & $24 \pm 10$ & $26 \pm 18$ & $20 \pm 14$ & 0.67 \\
CXR score & $6.2 \pm 1.4$ & $3.6 \pm 2.7$ & $0 \pm 0$ & $1.4 \pm 1.8$ & $<0.0001$ \\
PHYSIO & $17 \pm 8$ & $15 \pm 7$ & $13 \pm 9$ & $12 \pm 7$ & 0.20 \\
Exercise component & $18 \pm 11$ & $12 \pm 12$ & $14 \pm 11$ & $2 \pm 3$ & 0.0003 \\
CRP total & $52 \pm 18$ & $43 \pm 20$ & $38 \pm 23$ & $30 \pm 7$ & 0.04 \\
\hline
\end{tabular}

*: the following post hoc comparisons were significant at the $\mathrm{p}=0.05$ level; all other comparisons were nonsignificant: usual interstitial pneumonia (UIP) versus fibrotic nonspecific interstitial pneumonia (NSIP), cellular NSIP and respiratory bronchiolitis interstitial lung disease/desquamative interstitial pneumonia (RBILD/DIP) (chest radiographic (CXR) score); RBILD/DIP versus UIP and fibrotic NSIP (exercise component of the composite physiological score [7] (exercise component). See Clinical, radiological and physiological scoring section for details. UIP versus RBILD/DIP (CRP total score). ANOVA: analysis of variance; LOD: level of dyspnoea [7]; A-a: alveolar-arteriole oxygen gradient at rest; PHYSIO: composite physiological score (see Clinical, radiological and physiological scoring section for details).

analysis, the risk ratio (RR) of histological classification as UIP for mortality was $11.46(95 \%$ confidence interval (CI) 4.13-31.83; $\mathrm{p}<0.0001$ ). In a multivariate analysis adjusting for age, sex, smoking status, PHYSIO score, duration of symptoms and a CTfib score of $\geqslant 2$ in at least one lobe, the RR for mortality in the presence of UIP was 28.46 (95\% CI 5.47-147.98; $\mathrm{p}=0.0001)$. Importantly, female sex and a positive smoking history were protective. The RRs for these predictors are shown in table 4 . The results were similar when the analysis was confined to the UIP and NSIP cases.

UIP is a pathological diagnosis for which SLB is normally required. Whether or not a semiquantitative HRCT scoring system, previously shown to be predictive of mortality [6], could predict pathology was evaluated. The ability of a mean CTfib score of $\geqslant 2$ in any lobe (representing the presence of honeycomb change [10]) or mean CTfib score of $\geqslant 2$ for all lobes [6] to identify UIP was determined (table 5). This score was highly predictive for the presence of UIP. Importantly, a fibrotic score of $<2$

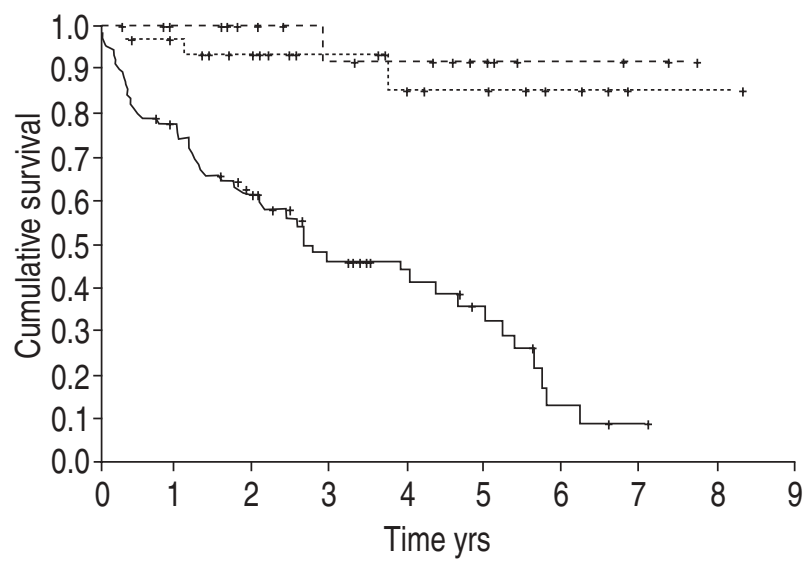

Fig. 1.- Kaplan-Meier survival curves in patients with usual interstitial pneumonia $(-, n=106)$, nonspecific interstitial pneumonia $(\cdots \cdots \cdots, \mathrm{n}=33)$ and respiratory bronchiolitis interstitial lung disease/ desquamative interstitial pneumonia $\left(-\right.$ - $\left.^{-}, \mathrm{n}=22\right)$ grouped by histological classification $(\mathrm{p}<0.00001)$. + : follow-up visits. did not exclude UIP. The results were similar when the analysis was confined to UIP and NSIP cases.

The CTfib score was predictive of subsequent

Table 4. - Risk ratios from Cox proportional hazard models predicting survival in patients with idiopathic interstitial pneumonia

\begin{tabular}{ll}
\hline Parameters in model & $\begin{array}{c}\text { Risk ratio } \\
(95 \% \mathrm{CI})\end{array}$ \\
\hline
\end{tabular}

\begin{tabular}{lll}
$\begin{array}{l}\text { Model excluding histological } \\
\text { subclassification }\end{array}$ & & \\
Patient age & $1.01(0.97-1.05)$ & 0.73 \\
Female sex & $0.53(0.25-1.14)$ & 0.11 \\
Positive smoking history & $0.43(0.18-1.01)$ & 0.05 \\
Physiological CRP score & $1.07(1.02-1.12)$ & 0.009 \\
Time of onset of symptoms & $1.04(0.96-1.14)$ & 0.32 \\
CTfib $\geqslant 2$ in at least one lobe & $3.35(1.21-9.25)$ & 0.02 \\
Model including histological & & \\
subclassification & & \\
UIP diagnosis & $28.46(5.47-147.98)$ & 0.0001 \\
Patient age & $0.99(0.95-1.03)$ & 0.55 \\
Female sex & $0.31(0.13-0.72)$ & 0.006 \\
Positive smoking history & $0.30(0.13-0.72)$ & 0.009 \\
Physiological CRP score & $1.06(1.01-1.11)$ & 0.02 \\
Time of onset of symptoms & $1.02(0.93-1.12)$ & 0.73 \\
CTfib $\geqslant 2$ in at least one lobe & $0.77(0.29-2.04)$ & 0.60 \\
\hline
\end{tabular}

CI: confidence interval; CRP: clinical, radiographical and physiological [score] [7]; CTfib: interstitial component of high-resolution computed tomography (see High-resolution computed tomography section for details); UIP: usual interstitial pneumonia.

Table 5. - Sensitivity, specificity, positive predictive value (PPV) and negative predictive value (NPV) of highresolution computed tomography fibrotic scores (CTfib) for identifying usual interstitial pneumonia in patients with idiopathic interstitial pneumonia

\begin{tabular}{lcccc}
\hline $\begin{array}{l}\text { Radiographic } \\
\text { feature }\end{array}$ & $\begin{array}{c}\text { Sensitivity } \\
\%\end{array}$ & $\begin{array}{c}\text { Specificity } \\
\%\end{array}$ & $\begin{array}{c}\text { PPV } \\
\%\end{array}$ & $\begin{array}{c}\text { NPV } \\
\%\end{array}$ \\
\hline $\begin{array}{l}\text { Mean CTfib } \geqslant 2 \text { for } \\
\text { all lobes }\end{array}$ & 46 & 93 & 93 & 47 \\
$\begin{array}{l}\text { Mean CTfib } \geqslant 2 \text { for } \\
\text { at least one lobe }\end{array}$ & 90 & 86 & 92 & 69 \\
\hline
\end{tabular}


mortality. The survival of patients with a CTfib score of $\geqslant 2$ in at least one lobe was worse than that of patients with a CTfib score of $<2$ in all lobes ( $\mathrm{p}=0.0001$ ) (fig. 2). A similar finding was noted when patients were segregated by mean CTfib of $\geqslant 2$ for all lobes $(\mathrm{p}=0.02)$. The RR for dying with a mean CTfib score of $\geqslant 2$ for the entire lung was 2.51 (95\% CI $1.39-4.54 ; \mathrm{p}=0.002)$, and 3.72 (95\% CI 1.83-7.56; $\mathrm{p}=0.0003)$ for those with at least one lobe with a CTfib score of $\geqslant 2$. A CTfib score of $\geqslant 2$ in at least
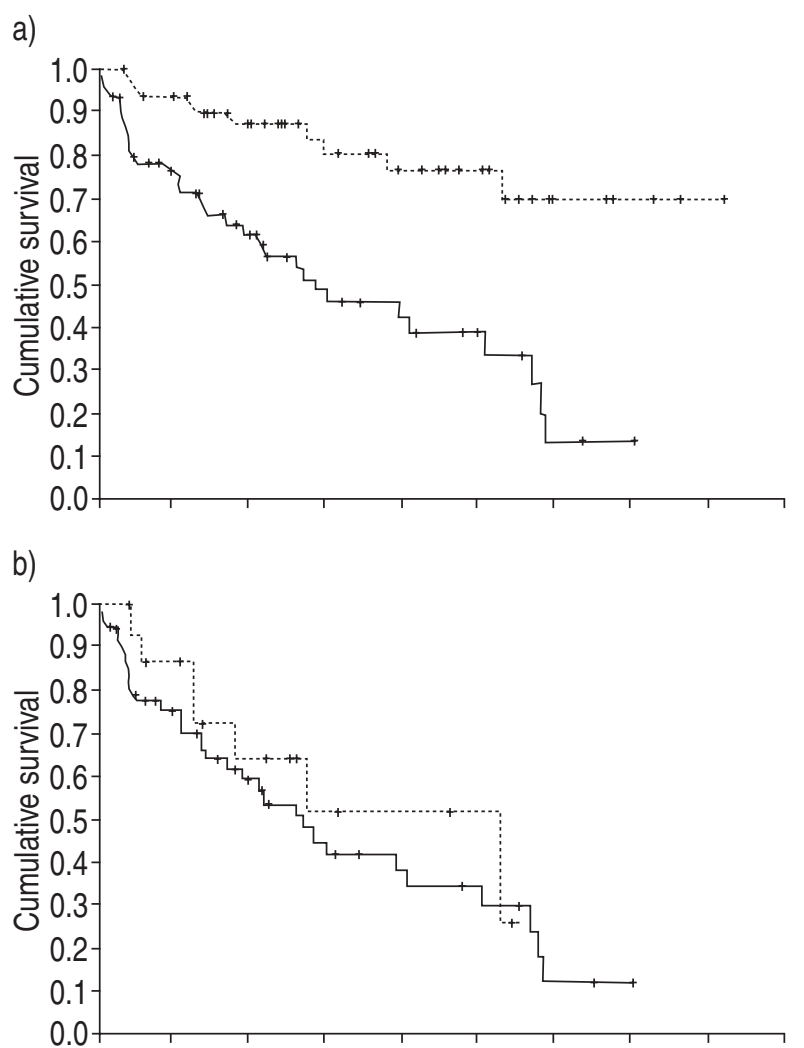

c)

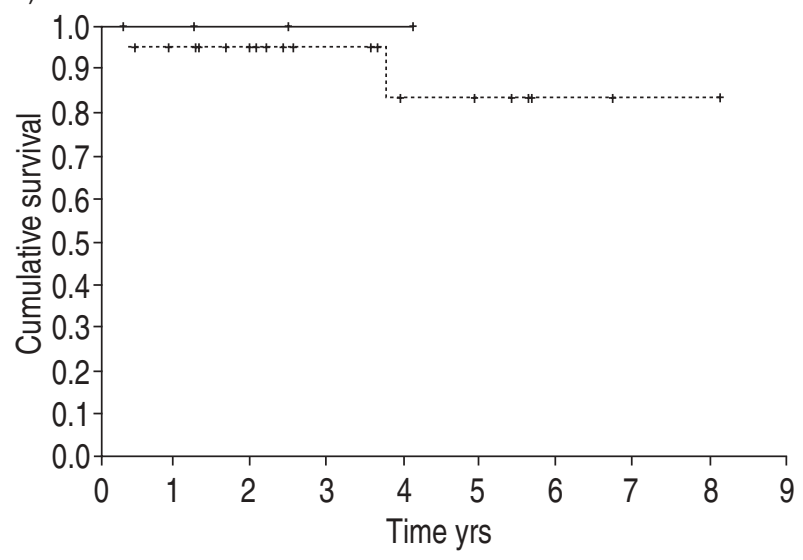

Fig. 2.-Kaplan-Meier survival curves in patients with: a) idiopathic interstitial pneumonia; b) usual interstitial pneumonia; and c) nonspecific interstitial pneumonia grouped by high-resolution computed tomography fibrotic score (CTfib) score of $\geqslant 2$ in at least one lobe $\left(\frac{}{2}\right.$, a) $n=72$, b) $n=66$, c) $\left.n=3\right)$ or no lobe with a CTfib score of $\geqslant 2(\cdots \cdots \cdots$, a) $n=54$, b) $n=17$, c) $n=24)$. a) $p<$ 0.00001 , b) $\mathrm{p}=0.47$, c) $\mathrm{p}=0.63$. + : follow-up visits. one lobe remained a significant predictor of mortality when taking into account patient age, sex, smoking history, PHYSIO score and time of onset of symptoms (table 4); a CTfib score of $\geqslant 2$ for the entire lung was not predictive in a similar multivariate model (RR 1.88; 95\% CI 0.83-4.26, p=0.13). When the histological diagnosis was placed in these models, it was the most powerful predictor of mortality and overshadowed the predictive ability of the CTfib score.

A RR for mortality in patients with different risk profiles can be calculated from these data. The following equation is based on the multivariate model presented in table 4.

$\mathrm{RR}=\frac{\mathrm{e}^{(3.35 \mathrm{a} 1-0.01 \mathrm{~b} 1-1.17 \mathrm{c} 1-1.20 \mathrm{~d} 1+0.06 \mathrm{e} 1+0.02 \mathrm{f} 1-0.26 \mathrm{~g} 1)}}{\mathrm{e}^{(3.35 \mathrm{a} 2-0.01 \mathrm{~b} 2-1.17 \mathrm{c} 2-1.20 \mathrm{~d} 2+0.06 \mathrm{e} 2+0.02 \mathrm{f} 2-0.26 \mathrm{~g} 2)}}$,

where $\mathrm{a}$ is histology ( $1=\mathrm{UIP}, 0=$ other $), \mathrm{b}$ is age, $\mathrm{c}$ is sex ( $1=$ male, $0=$ female), $d$ is smoking $(1=$ smoker, $0=$ nonsmoker), e is physiological component of the CRP score, $\mathrm{f}$ is time of onset of symptoms, $\mathrm{g}$ is CTfib score $(1=$ score $\geqslant 2$ in at least one lobe, $0=$ score $<2$ in all lobes) and the 1 and 2 denote patients 1 and 2 . The physiological component of the CRP score, age and onset of symptoms in years are entered as continuous variables. The physiological component of the CRP score incorporates the scores for pulmonary function and resting oxygenation (see Methods section). For example, the RR for mortality in patient No. 112 (UIP histology, 78 yrs old, male sex, nonsmoker, physiological component of CRP score 26, onset of symptoms $1 \mathrm{yr}$ and lobar CTfib score $\geqslant 2$ ) is 154 times higher than that of patient No. 123 (NSIP histology, 49 yrs old, female sex, smoker, physiological component of CRP score 11, onset of symptoms $1.5 \mathrm{yrs}$ and no lobar CTfib score $\geqslant 2$ ). Patient No. 112 died after $1.0 \mathrm{yr}$, whereas patient No. 123 remains alive after 4.0 yrs.

The treatment regimens for patients with UIP and NSIP were heterogeneous, as enumerated in table 1. No consistent survival effect was noted for any therapeutic regimen, although detailed analysis was precluded by differences in treatment protocols and the lack of a predefined response to therapy. Patients (39), characterized as UIP or NSIP using the latest histopathological criteria, were treated prospectively with high-dose prednisone using CRP score to define their response (fig. 3). Patients with NSIP $(n=10)$ included more responders (40 versus $17 \%$ ) and fewer nonresponders (10 versus 52\%) compared to patients with UIP ( $p=0.05$, Fisher exact test). Patients $(n=39)$ who remained stable after an initial 3-month course of therapy exhibited improved survival (RR compared to nonresponders 0.32 ; $95 \%$ CI $0.11-0.93$; $\mathrm{p}=0.04)$. This effect persisted after accounting for histology, age, sex and smoking history. This survival advantage was seen when patients with UIP were analysed separately (RR for stability compared to nonresponders 0.33 ; 95\% CI 0.12-0.96; $p=0.04$ ) (fig. 4). No difference was seen in baseline clinical, physiological or radiographic features between UIP patients segregated by their subsequent response to prednisone therapy. Only one patient died in the subset of NSIP patients treated with high-dose prednisone, precluding further analysis of this group. The baseline characteristics between 


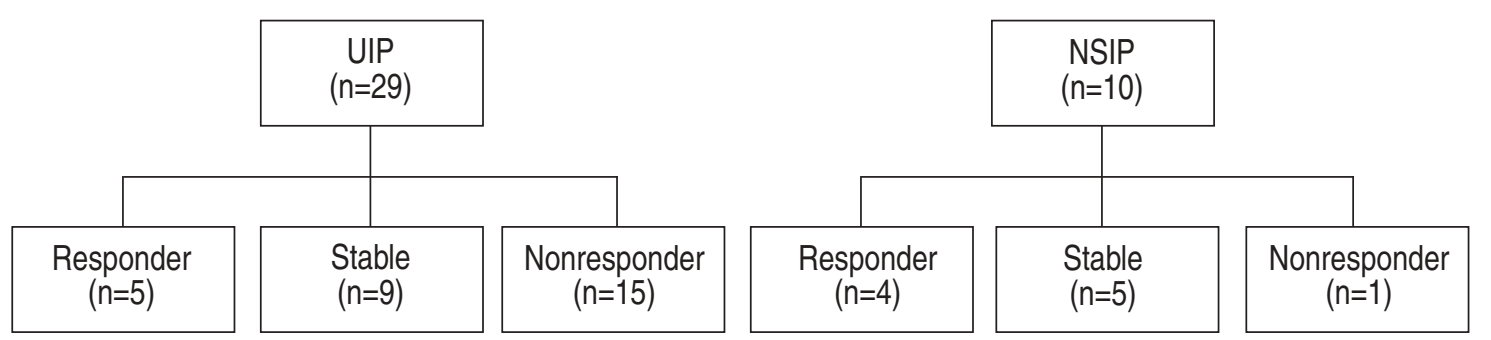

Fig. 3. - Results of high-dose steroid therapy in 29 patients with usual interstitial pneumonia (UIP) and 10 with nonspecific interstitial pneumonia (NSIP). Response to therapy was assessed by change in clinical, radiological and physiological (CRP) score after 3 months of therapy. Responders were defined as having a $>10$-point drop in CRP score, stable a \pm 10 change in CRP score and nonresponders a $>10$-point rise in CRP score [6, 7]. A difference in the distribution of response was seen between UIP and NSIP patients ( $p=0.05$, Fisher exact test).

UIP patients treated as part of the prospective prednisone trial were similar to those not treated in this protocol with the exception of having a lower CRP score $(47 \pm 16$ versus $56 \pm 18$; $\mathrm{p}=0.05)$, lower CTalv score $(1.58 \pm 0.81$ versus $2.01 \pm 0.89 ; \mathrm{p}=0.03)$, higher CTfib score $(2.12 \pm 0.61$ versus $1.76 \pm 0.74 ; \mathrm{p}=0.02)$ and higher BDI $(6.7 \pm 2.7$ versus $5.0 \pm 2.2 ; \mathrm{p}=0.01)$.

\section{Discussion}

In the present report of a large number of patients with IIP, the following are quantified: 1) the paramount importance of a histological diagnosis of UIP in predicting mortality; 2) the sensitivity and specificity of HRCT scoring for diagnosing UIP; and 3) the response to treatment with high-dose prednisone for patients treated prospectively using a standardized protocol. A pathological diagnosis of NSIP was made in $\sim 20 \%$ of patients referred to a tertiary referral centre with IIP and RBILD/DIP was seen in $\sim 13 \%$. These percentages are similar to those reported by others [11, 13-15]. These data aid in defining the patient population most likely to require SLB for

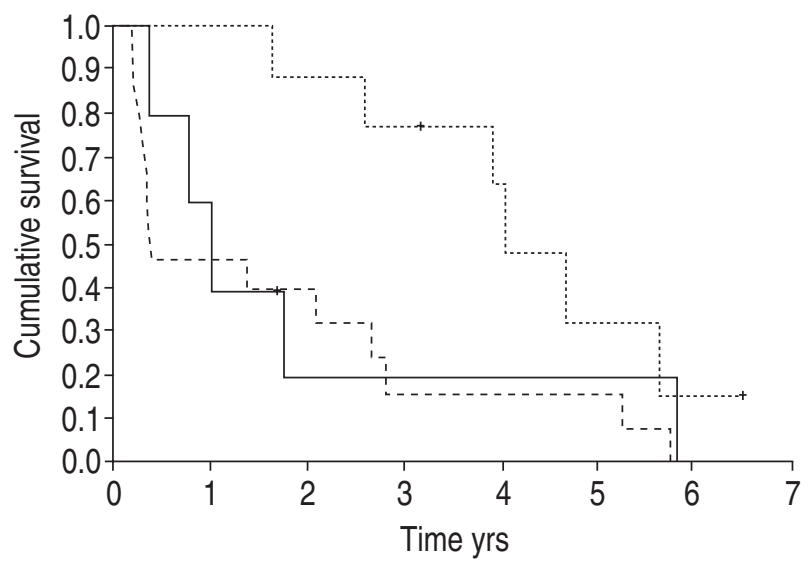

Fig. 4.-Kaplan-Meier survival curves in patients with usual interstitial pneumonia $(n=29)$ treated with high-dose steroids for 3 months grouped by response status as defined by change in clinical, radiological and physiological (CRP) score at 3 months $(\mathrm{p}=0.02)$. Responders were defined as having a $>10$-point drop in CRP score $(-)$, stable a \pm 10 change in CRP score $(\cdots \cdots \cdots \cdot)$ and nonresponders a $>10$-point rise in CRP score (---). +: follow-up visits. definitive diagnosis and which patients are most likely to benefit from prednisone therapy.

The present data document that a histological diagnosis of UIP is associated with a nearly 30 -fold greater risk of mortality than an alternative histological diagnosis in patients evaluated for suspected IIP. Furthermore, the RR of mortality for a histological diagnosis of UIP was more than 10 times higher than that associated with the presence of honeycomb change on HRCT. The design of this study strengthens these findings. All standard clinical indices (age, sex, smoking history and duration of symptoms before SLB), physiological studies and HRCT scores were prospectively acquired. All pathological specimens were reviewed by three pathologists using the latest criteria to define histopathological subsets $[2,3,11]$.

Obtaining a histological diagnosis of UIP requires SLB, which is not feasible in all patients, and is infrequently performed in clinical practice $[2,16]$. The presence of honeycombing on HRCT (indicated by a CTfib score of $\geqslant 2$ ) is a powerful predictor of survival when the histological diagnosis is unknown. The different RRs of lobar versus whole lung CTfib score probably reflect the presence of disease heterogeneity. Although the magnitude of risk of a radiographic diagnosis is less than that of a histological diagnosis of UIP, it can be useful for patients that are unable to undergo SLB. It also suggests that the presence of honeycombing may be a surrogate marker for UIP.

UIP patients demonstrated greater fibrotic abnormality on HRCT than NSIP and RBILD/DIP patients. A wide variety of radiographic patterns have been described in NSIP [17-21]. In the current series, a CTfib score of $\geqslant 2$ in at least one lobe had a high positive predictive value for the presence of UIP on SLB. The excellent positive predictive value suggests that these patients do not need to undergo SLB for clarification of diagnosis. Semiquantitative HRCT scores are independent of a radiologist's interpretation of the pattern seen on HRCT and exhibit good interobserver agreement among expert radiologists [10].

The negative predictive value of a CTfib score of $\geqslant 2$ was limited. This reflects the absence of honeycombing on HRCT in many patients with UIP. Interestingly, the negative predictive value was similar to 
that previously reported for a nonquantitative expert HRCT interpretation [22]. Since a semiquantitative CTfib score of $<2$ is not reliably predictive of the absence of UIP, the present data argue strongly for the performance of SLB in the diagnostic evaluation of these patients. Importantly, this represented $43 \%$ of UIP patients in this study.

Female sex and a positive smoking history were associated with improved survival, even in the presence of UIP. The magnitude of the protective effect of female sex was similar to that previously reported [23] and much smaller than the increased mortality associated with a UIP diagnosis. Importantly, improved survival was not noted in younger patients, which may reflect the younger age of patients with non-UIP histological diagnoses.

The present data are the first to quantify a protective effect associated with smoking. The cause of this protective effect is unclear, but could be an inhibitory effect of cigarette smoke on fibroblast function [24]. Interestingly, no difference in smoking history was noted between patients with UIP, NSIP and RBILD/DIP. Although early reports suggested that RBILD/DIP was seen exclusively in smokers [25], recent reports have suggested that a significant minority of cases may be seen in nonsmokers $[15,26]$. This difference could indicate that RBILD/DIP may be seen without smoking, although the possibility that the present patients did not disclose their smoking history also exists. Further research into the role of cigarette smoking and the pathogenesis of interstitial lung disease is needed.

Patients whose CRP scores remained stable following 3 months of prednisone therapy experienced improved survival compared to those who responded or failed to respond to an initial course of prednisone. This finding was noted in the entire group and in UIP patients. The protective effect of stability following prednisone therapy was of a similar magnitude to that of female sex and smoking history. Importantly, UIP patients who remained stable during prednisone therapy could not be distinguished from those who were responders or nonresponders using the baseline data. This stability during initial therapy might reflect the effect of treatment, the effect of lead time bias or be the result of a different natural history in a subgroup of UIP patients that is independent of therapy. Future trials with prospective randomized controlled data collection are required to define the effect of therapy on mortality. There was a modest increase in mortality associated with increased physiological abnormality, as reflected by the physiological component of the CRP score. These data suggest that the degree of physiological abnormality at the time of evaluation has predictive value, although the RR is quite modest in comparison to the impact of histological diagnosis and radiographic abnormality.

The risk ratios provided by the above data allow for a quantitative approach to predicting the likelihood of mortality in different clinical situations for patients with idiopathic interstitial pneumonia. The log transformation of risk ratio can be utilized to compare survival in two divergent clinical scenarios. The present study also provides support for a stepwise diagnostic approach to new patients with idiopathic interstitial pneumonia. Given that semiquantitative high-resolution computed tomography scoring provides valuable diagnostic information, all patients suspected of having idiopathic interstitial pneumonia should undergo this technique. The presence of an interstitial component of high-resolution computed tomography score of $\geqslant 2$ reliably identifies patients with usual interstitial pneumonia. Surgical lung biopsy is warranted for patients without honeycombing on high-resolution computed tomography; the absence of honeycombing does not exclude a pathological diagnosis of usual interstitial pneumonia. In the current group, this diagnostic approach would have resulted in a recommendation for surgical lung biopsy in 43\% (54 of 126) of patients; in those patients in whom a biopsy would not be recommended, only $8 \%$ (6 of 72 ) would have been misclassified as having usual interstitial pneumonia. The present study documents that a noninvasive diagnostic procedure, high-resolution computed tomography, can aid in the diagnosis of usual interstitial pneumonia in a significant proportion of patients with idiopathic interstitial pneumonia. Accordingly, these patients could be spared the cost and risk of surgical lung biopsy. However, the presence of usual interstitial pneumonia on surgical lung biopsy remains the most powerful predictor of mortality in patients with idiopathic interstitial pneumonia.

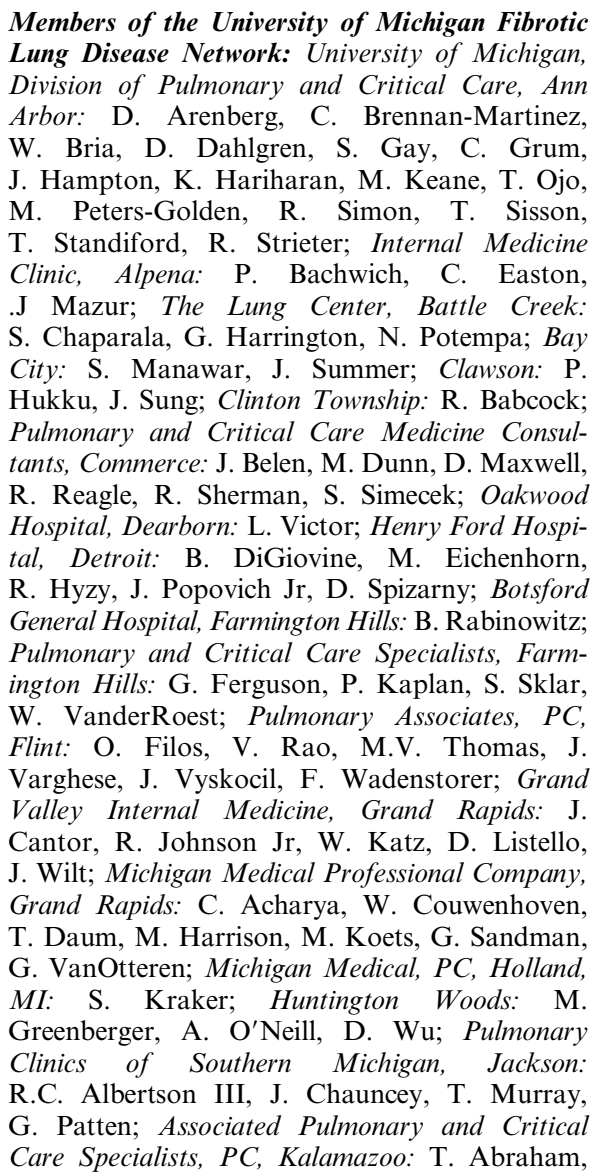


J. Dirks, B. Dykstra, G. Grambau, J. Schoell; Pulmonary and Critical Care Associates, PC, Kalamazoo: R. Brush, S. Jefferson, J. Miller, S. Schuldheisz, M. Warlick; Pulmonary and Critical Care Consultants, Lansing: J. Armstrong, A. Atkinson, T. Kantra, L. Rawsthorne, D. Young; Pulmonary Services, Lansing: A. Abbasi, C.M. Gera, G. Kashyap, J. Morlock; Respiratory Medicine, Marquette, MI; S. Danek, A. Saari; Midland: S. Yadam; Central Michigan Healthcare System, Mt. Pleasant: E. Obeid; Muskegon Pulmonary Associates, Muskegon: D. Hoch, A. Kleaveland; Owosso Medical Group, Owosso: A. Allam, M.A. Gad Jr; Lung Associates, Pontiac: A. Desai, U. Dhanjal, A. Sethi; St Joseph's Hospital, Pontiac: F. Ahmad, R. Elkus, L. Kaiser, L. Rosenthal, D. Sak; Physician HealthCare Network, Port Huron: R. Ailani, M. Basha, A. Hadar, S. Holstine; Rochester: K. Aggarwal; Pulmonary, Critical Care, and Sleep, PC, Rochester Hills: M.W. Al-Ameri, R. Go, M. Kashlan; Roseville: W. Hanna, R. Marchese; William Beaumont Hospital, Royal Oak: R. Begle, D. Erb, K.P. Ravikrishnan, J. Seidman, S. Sherman; Saginaw: R. Agarwal, F. Ansari, T. Damuth, C. Indira; Pulmonary and Critical Care Associates, St Clair Shores and Troy: R. DiLisio, S. Galens, K. Grady, D. Harrington, R. Herbert, C. Hughes, J. Lee, A. Starrico, K. Stevens, M. Trunsky, W. Ventimiglia; Lakeside Healthcare Specialists, St Joseph: S. Deskins, A. Palmer, S. Shastri; Spring Lake, MI: M. Ivey; Taylor: D. Mahajan; Pulmonary Medicine Associates, Warren: H. Kaplan, L. Tankanow; Henry Ford Wyandotte Hospital, Wyandotte: M. Pensler; Toledo Pulmonary and Sleep Specialists, Toledo, $O H$, USA: F.O. Horton III, A. Nathanson, R. Wainz (all in MI, USA, unless otherwise indicated).

\section{References}

1. American Thoracic Society. Idiopathic pulmonary fibrosis: diagnosis and treatment. International consensus statement. Am J Respir Crit Care Med 2000; 161: 646-664.

2. Katzenstein ALA, Myers JL. Idiopathic pulmonary fibrosis. Clinical relevance of pathologic classification. Am J Respir Crit Care Med 1998; 157: 1301-1315.

3. Ryu JH, Colby TV, Hartman TE. Idiopathic pulmonary fibrosis: current concepts. Mayo Clin Proc 1998; 73: 1085-1101.

4. Flint A, Martinez FJ, Young ML, Whyte RI, Toews GB, Lynch JP III. Influence of sample and number and biopsy site on the histologic diagnosis of diffuse lung disease. Ann Thorac Surg 1995; 60: 1607-1608.

5. Flaherty KR, Toews GB, Lynch JP III, et al. Steroids in idiopathic pulmonary fibrosis: a prospective assessment of adverse reactions, response to therapy, and survival. Am J Med 2001; 110: 278-282.

6. Gay SE, Kazerooni EA, Toews GB, et al. Idiopathic pulmonary fibrosis. Predicting response to therapy and survival. Am J Respir Crit Care Med 1998; 157: $1063-1072$.

7. Watters LC, King TE, Schwarz MI, Waldron JA, Stanford RE. A clinical, radiographic, and physiologic scoring system for the longitudinal assessment of patients with idiopathic pulmonary fibrosis. Am Rev Respir Dis 1986; 133: 97-103.

8. Mahler D, Weinberg D, Wells C, Feinstein A. The measurement of dyspnea: contents, interobserver agreement, and physiologic correlates of two new clinical indices. Chest 1984; 85: 751-758.

9. McGravin CR, Artvinli M, Naoe H. Dyspnoea, disability, and distance walked: comparison of exercise performance in respiratory disease. BMJ 1978; 2: 241243.

10. Kazerooni EA, Martinez FJ, Flint A, et al. Thinsection CT obtained at $10 \mathrm{~mm}$ increments versus three-level thin-section CT for idiopathic pulmonary fibrosis: correlation with pathologic scoring. $\mathrm{Am}$ J Roentgenol 1997; 169: 977-983.

11. Travis WD, Matsui K, Moss J, Ferrans VJ. Idiopathic nonspecific interstitial pneumonia: prognostic significance of cellular and fibrosing patterns. Am J Surg Path 2000; 24: 19-33.

12. Katzenstein ALA, Fiorelli RF. Nonspecific interstitial pneumonia/fibrosis. Histologic features and clinical significance. Am J Surg Path 1994; 18: 136-147.

13. Daniil ZD, Gilchrist FC, Nicholson AG, et al. A histologic pattern of nonspecific interstitial pneumonia is associated with a better prognosis than usual interstitial pneumonia in patients with cryptogenic fibrosing alveolitis. Am J Respir Crit Care Med 1999; 160: 899-905.

14. Bjoraker JA, Ryu JH, Edwin MK, et al. Prognostic significance of histopathologic subsets in idiopathic pulmonary fibrosis. Am J Respir Crit Care Med 1998; 157: 199-203.

15. Nicholson AG, Colby TV, DuBois RM, Hansell DM, Wells AU. The prognostic significance of the histologic pattern of interstitial pneumonia in patients presenting with the clinical entity of cryptogenic fibrosing alveolitis. Am J Respir Crit Care Med 2000; 162: 2213-2217.

16. Johnston IDA, Prescott RJ, Chalmers JC, Rudd RM. British Thoracic Society study of cryptogenic fibrosing alveolitis: current presentation and initial management. Thorax 1997; 52: 38-44.

17. Johkoh T, Muller NL, Cartier Y, et al. Idiopathic interstitial pneumonias: diagnostic accuracy of thinsection CT in 129 patients. Radiology 1999; 211: 555560 .

18. Hartman TE, Swensen SJ, Hansell DM, et al. Nonspecific interstitial pneumonia: variable appearance at high-resolution chest CT. Radiology 2000; 217: 701705.

19. Kim TS, Lee KS, Chuang MP, et al. Nonspecific interstitial pneumonia with fibrosis: high-resolution CT and pathologic findings. Am JRoentgenol 1998; 171: 1645-1650.

20. Park JS, Lee KS, Kim JS, et al. Nonspecific interstitial pneumonia with fibrosis: radiographic and CT findings in seven patients. Radiology 1995; 195: 645-648.

21. Nagai S, Kitaichi M, Itoh H, Nishimura K, Izumi T, Colby TV. Idiopathic nonspecific interstitial pneumonia/fibrosis: comparison with idiopathic pulmonary fibrosis and BOOP. Eur Respir J 1998; 12: 1010-1019.

22. Raghu G, Mageto YN, Lockhart D, Schmidt RA, Wood DA, Godwin JD. The accuracy of the clinical diagnosis of new-onset idiopathic pulmonary fibrosis and other interstitial lung disease. A prospective study. Chest 1999; 116: 1168-1174.

23. Douglas WW, Ryu JH, Schroeder DR. Idiopathic 
pulmonary fibrosis: impact of oxygen and colchicine, prednisone, or no therapy on survival. Am J Respir Crit Care Med 2000; 161: 1172-1178.

24. Carnevali S, Nakamura Y, Mio T, et al. Cigarette smoke extract inhibits fibroblast-mediated collagen gel contraction. Am J Physiol 1998; 274: L591-L598.

25. Ryu JH, Colby TV, Hartman TE, Vassallo R.
Smoking-related interstitial lung diseases: a concise review. Eur Respir J 2001; 17: 122-132.

26. Moon J, DuBois RM, Colby TV, Hansell DM, Nicholson AG. Clinical significance of respiratory bronchiolitis on open lung biopsy and its relationship to smoking related interstitial lung disease. Thorax 1999; 54: 1009-1014. 